\title{
Millimeter- and submillimeter-wave spectrum of trans-formaldoxime $\left(\mathrm{CH}_{2} \mathrm{NOH}\right)^{\star}$
}

\author{
Luyao Zou ${ }^{1}$, Jean-Claude Guillemin ${ }^{2}$, Roman A. Motiyenko ${ }^{1}$, and Laurent Margulès ${ }^{1}$ \\ ${ }^{1}$ Univ. Lille, CNRS, UMR 8523 - PhLAM - Physique des Lasers Atomes et Molécules, 59000 Lille, France \\ e-mail: luyao.zou@univ-lille.fr \\ ${ }^{2}$ Univ. Rennes, Ecole Nationale Supérieure de Chimie de Rennes, CNRS, ISCR-UMR 6226, 35000 Rennes, France
}

Received 16 December 2020 / Accepted 10 March 2021

\begin{abstract}
Context. Among the six atoms of $\mathrm{N}$-containing molecules with the formula of $\mathrm{CH}_{3} \mathrm{NO}$, only formamide $\left(\mathrm{H}_{2} \mathrm{NCHO}\right)$, the most stable structural isomer, has been detected in the interstellar medium (ISM). The formaldoxime isomer may be formed, for example, by the reaction of formaldehyde $\left(\mathrm{H}_{2} \mathrm{CO}\right)$ or methanimine $\left(\mathrm{H}_{2} \mathrm{CNH}\right)$ and hydroxylamine $\left(\mathrm{H}_{2} \mathrm{NOH}\right)$, which are all detected in the ISM. The lack of high accuracy millimeter- and submillimeter-wave measurements hinders the astronomical search for formaldoxime.

Aims. The aim of this work is to provide the direct laboratory measurement of the millimeter- and submillimeter-wave spectrum of trans-formaldoxime.

Methods. Formaldoxime was synthesized and its rotational spectrum was recorded at room temperature in a glass flow cell using the millimeter- and submillimeter-wave spectrometer in Lille. The SPFIT program in the CALPGM suite was used to fit the spectrum.

Results. Rotational lines of trans-formaldoxime from both the ground state and $v_{12}=1$ vibrational excited states have been measured and assigned from 150 to $660 \mathrm{GHz}$. Spectroscopic constants were derived to the tenth order using both Watson's A and S reduction Hamiltonian.
\end{abstract}

Key words. astrochemistry - molecular data - submillimeter: ISM - methods: laboratory: molecular

\section{Introduction}

Of the little more than 200 molecules detected in the interstellar medium (ISM) (McGuire 2018), about 20 compounds with six atoms constitute the series of the smallest complex organic molecules (COMs) (Herbst \& van Dishoeck 2009). This is the most abundant group among COMs, an observation consistent with the fact that the number of molecules detected decreases with the number of atoms.

To find new compounds in the ISM, the choice of new candidates is mainly based on the analogy with the molecules detected, the possible chemistry of each interstellar cloud, and the physical parameters such as the relative thermodynamic stability of the isomers. Many detected compounds have an isomer in the list (McGuire 2018).

Among the six-atom molecules, only one isomer with a $\mathrm{CH}_{3} \mathrm{NO}$ formula has been detected, the formamide, $\mathrm{H}_{2} \mathrm{NCHO}$, which was found in Sgr B2 and Sgr A in 1971 (Rubin et al. 1971). The following isomers in relative thermodynamic stability are the tautomer $\mathrm{C}$-hydroxymethanimine (methanimidic acid, $\mathrm{HN}=\mathrm{CHOH}$ ), which is $13.4 \mathrm{kcal} \mathrm{mol}^{-1}$ less stable than formamide, and formaldoxime $\left(\mathrm{CH}_{2} \mathrm{NOH}\right)$, which is $51.0 \mathrm{kcal} \mathrm{mol}^{-1}$ less stable than formamide (Lattelais et al. 2010). The observation of methanimine, a reduced form of formaldoxime in the molecular cloud Sgr B2 (Godfrey et al. 1973), and the recent detection of hydroxylamine, $\mathrm{H}_{2} \mathrm{NOH}$, in the quiescent molecular cloud G+0.693-0.027 located in the Galactic Center (Rivilla et al. 2020) prompted us to study formaldoxime.

${ }^{\star}$ Full Tables 2, 3, A.1, A.2 are only available at the CDS via anonymous ftp to cdsarc.u-strasbg.fr (130.79.128.5) or via http: //cdsarc.u-strasbg.fr/viz-bin/cat/J/A+A/649/A60
Methanimine is possibly synthesized in the ISM by the reaction of formaldehyde with ammonia (Vinogradoff et al. 2011), while the reaction of formaldehyde with hydroxylamine could form formaldoxime. The very reactive methanimine has never been prepared on a gram-scale in the laboratory in this way, but such an approach has been used since the end of the 19th century to prepare formaldoxime (Dunstan \& Bossi 1898). Formaldoxime could also be formed in the ISM in other ways such as the oxidation of methanimine $\left(\mathrm{H}_{2} \mathrm{CNH}\right)$ or the reaction of the latter with $\mathrm{H}_{2} \mathrm{NOH}$. To our knowledge, attempts to form formaldoxime by simulating the chemistry on the grains or in the gas phase of the ISM have never been carried out. However, as an isomer of the detected formamide, on the basis of a possible formation in the ISM starting from detected compounds, and by analogy with the imines observed in this medium, formaldoxime appears to be a good candidate as a component of interstellar clouds.

Formaldoxime is a prolate-type asymmetric top rotor. The microwave spectrum of formaldoxime was first reported by Levine (1962) and by Pillai (1962) independently. The structure revealed by isotopologue substitution suggests that this molecule has a planar trans geometry, with a small dipole moment: 0.40 Debye for $a$-dipole and 0.18-0.20 Debye for $b$ dipole (Levine 1962, 1963). The more stable trans isomer was supported by an ab initio calculation, which shows that the cis isomer has a significantly larger dipole moment ( $>3$ Debye), and it lies about $5.6 \mathrm{kcal} \mathrm{mol}^{-1}$ above the trans isomer (Umar et al. 2005). From this energy perspective, the cis isomer has a Boltzmann population ratio of only $78 \mathrm{ppm}$ under room temperature. Although in the ISM, the possibility of kinetic processes that can pump the cis isomer to a higher population cannot be ruled out, in this article, we only focus on the trans isomer, and 
therefore the term "trans" is not used hereafter. Kaushik \& Takagi (1978) reanalyzed the spectra of Levine (1962) to show the strong centrifugal distortion effects of this molecule up to the quartic order. In the studies by Levine and Pillai, the frequency resolution was $\sim 0.1 \mathrm{MHz}$. The microwave spectrum with a much higher frequency resolution $(\sim 2 \mathrm{kHz})$ was measured by Klesing \& Sutter (1990) to fully resolve the splitting due to the ${ }^{14} \mathrm{~N}$ nuclear spin. Rotationally-resolved infrared spectra of formaldoxime were reported across the infrared band from $30 \mu \mathrm{m}$ to $1.5 \mu \mathrm{m}$ (Duxbury et al. 1988; Duxbury 1988; Bannai \& Duxbury 1994; Wu \& Tan 2020, 2021). Several submillimeter-wave laser transitions of formaldoxime, pumped by $\mathrm{CO}_{2}$ lasers, were also reported. The frequencies, however, were not well determined because only four significant figures were reported in the unit of micron (Duxbury \& Petersen 1984).

Due to the limited frequency resolution in the infrared and the limited numbers of detectable transitions in the microwave, uncertainties still remain in the spectroscopic constants of formaldoxime, and therefore in its predicted transition frequencies in the millimeter- and submillimeter-wave range. To date, the most accurate centrifugal distortion analysis has been performed by $\mathrm{Wu} \&$ Tan $(2020,2021)$ using a high resolution synchrotron infrared beamline. In their studies, the highest $J$ and $K_{\mathrm{a}}$ values accessed are 45 and 15 , respectively. The complete set of five quartic terms, as well as three sextic terms, were determined. Their frequency measurement has an uncertainty of $\sim 6 \mathrm{MHz}\left(0.0002 \mathrm{~cm}^{-1}\right)$, and their fit has a root-mean-square (r.m.s.) of $\sim 15 \mathrm{MHz}\left(0.0005 \mathrm{~cm}^{-1}\right)$. Nevertheless, this accuracy is still insufficient for the astronomical search of the millimeterand submillimeter-wave lines of formaldoxime in the ISM. In this article, we report the experimental measurement of the millimeter- and submillimeter-wave direct absorption spectrum of formaldoxime between $150-330 \mathrm{GHz}$ and $400-660 \mathrm{GHz}$. The high resolution data allow us to fully characterize the centrifugal distortion effects of formaldoxime up to the decic order, which is sufficiently accurate for astronomical searches.

\section{Experimental methods}

\subsection{Synthesis of formaldoxime}

The synthesis of Dunstan and Bossi has been modified (Dunstan \& Bossi 1898). A 33\% aqueous solution of formaldehyde was prepared by stirring two masses of water with one mass of paraformaldehyde for $1 \mathrm{~h}$ at $80{ }^{\circ} \mathrm{C}$. The mixture was then cooled to room temperature and one equivalent of hydroxylamine, hydrochloride and 0.5 equivalent of sodium carbonate were added. After stirring overnight, the evaporation of the water and low boiling compounds by heating to $60{ }^{\circ} \mathrm{C}$ under vacuum gives a solid containing a mixture of the trimer with sodium chloride. By heating this solid under vacuum to $100{ }^{\circ} \mathrm{C}$, a substantially pure gaseous flow of formaldoxime was obtained. The ${ }^{1} \mathrm{H}$ NMR and ${ }^{13} \mathrm{C}$ NMR results of the sample are the following: ${ }^{1} \mathrm{H} \mathrm{NMR}\left(\mathrm{CDCl}_{3}, 400 \mathrm{MHz}\right) \delta 6.48\left(\mathrm{~d},{ }^{2} \mathrm{~J}_{\mathrm{HH}}=8.5 \mathrm{~Hz}\right) ; 7.05$ $\left(\mathrm{d},{ }^{2} J_{\mathrm{HH}}=8.5 \mathrm{~Hz}\right) ; 8.2-9.6(\mathrm{brd}, 1 \mathrm{H}, \mathrm{OH}) ;{ }^{13} \mathrm{C} \mathrm{NMR}\left(\mathrm{CDCl}_{3}\right.$, $100 \mathrm{MHz}) \delta 138.8\left(\mathrm{dd},{ }^{1} J_{\mathrm{CH}}=180.4 \mathrm{~Hz}\right.$ and $\left.167.2 \mathrm{~Hz}\right)$.

\subsection{Millimeter- and submillimeter-wave spectrum of formaldoxime}

The millimeter- and submillimeter-wave spectrum of $\mathrm{CH}_{2} \mathrm{NOH}$ was measured using the fast absorption spectrometer in Lille (Zakharenko et al. 2015; Motiyenko et al. 2019). In brief, the $\mathrm{CH}_{2} \mathrm{NOH}$ vapor was introduced into a $2-\mathrm{m}$ absorption cell by
Table 1. Multiplier specification and experimental conditions.

\begin{tabular}{ccccc}
\hline \hline Band & Harmonics & $\begin{array}{c}\text { Frequency } \\
\text { range }(\mathrm{GHz})\end{array}$ & $\begin{array}{c}\text { Step } \\
(\mathrm{kHz})\end{array}$ & $\begin{array}{c}\text { Pressure } \\
(\mu \mathrm{bar})\end{array}$ \\
\hline WR5.1 & $\times 12$ & $150-220$ & 36 & $26-36$ \\
WR3.4 & $\times 18$ & $225-330$ & 42 & $40-52$ \\
WR1.9 & $\times 30$ & $400-480$ & 60 & $63-68$ \\
WR1.5 & $\times 36$ & $480-660$ & 72 & $63-68$ \\
\hline
\end{tabular}

heating the solid trimer mixture. After filling the cell with $\mathrm{CH}_{2} \mathrm{NOH}$ gas to a decent pressure, the cell was left static for measurement. The millimeter- and submillimeter-wave radiation was generated by a series of frequency multipliers (Virginia Diodes, Inc.), which were pumped by $12-18 \mathrm{GHz}$ radio frequency generated by a synthesizer (Agilent, E8257), and detected by a hot electron bolometer (QMC). We used the sine-wave frequency modulation and second harmonic detection technique to improve the signal-to-noise ratio. The modulation frequency was set at $20.5 \mathrm{kHz}$ and the deviation was set at $15 \mathrm{kHz}$. The time constant of the lock-in amplifier (Metek 7270 DSP) was $200 \mu \mathrm{s}$ and the integration time was $1 \mathrm{~ms}$. The spectra between $150 \mathrm{GHz}$ and $660 \mathrm{GHz}$ were covered by four frequency bands. The frequency multiplication, frequency range, scanning step, and sample pressure are summarized in Table 1 . The signalto-noise ratio was sufficiently high to allow two averages for the whole frequency range. For a few extremely weak features, 8-16 averages were measured in a small frequency window around the lines.

The line frequencies were obtained by fitting the second derivative of the Voigt profile. The estimated uncertainty on transition frequencies ranges from $30 \mathrm{kHz}$ to $100 \mathrm{kHz}$, depending on the signal-to-noise ratio of the line, the selection rule, and the splitting of the hyperfine structure. In brief, fully resolved lines were assigned with a smaller frequency uncertainty than blended lines, and $a$-type transitions were assigned with a smaller frequency uncertainty than $b$-type transitions, because $b$-type transitions are not only weaker due to the smaller $b$ dipole moment, but they also have larger hyperfine splitting, which becomes only partially resolvable in the millimeter- and submillimeterwave range. The complete list of observed lines, as well as their estimated uncertainty, can be found in CDS: S1-S2.

The spectral lines of $\mathrm{CH}_{2} \mathrm{NOH}$ were fit using the SPFIT program in the CALPGM suite (Pickett 1991). Partially blended lines were weighted by their relative intensities.

The ab initio ${ }^{14} \mathrm{~N}$ quadrupole hyperfine splitting constants and the dipole moment of $\mathrm{CH}_{2} \mathrm{NOH}$ were calculated using the Gaussian09 software (Frisch et al. 2016). Geometry was optimized using the B3LYP level of theory and 6-311++g(3df,2pd) basis set. The optimized geometry was used in single point energy calculation, using the B3PW91 level of theory and 6-311+G(df,pd) basis set.

\section{Results and discussion}

Before the spectral assignment, the standing waves were removed from the millimeter- and submillimeter-wave spectrum of $\mathrm{CH}_{2} \mathrm{NOH}$, and peaks were identified and fit to obtain the line frequencies. In the spectrum, we observed contamination lines from acetonitrile $\left(\mathrm{CH}_{3} \mathrm{CN}\right)$, which possibly originated from the residual on our vapor inlet. The contamination lines consist of those from the ground state of $\mathrm{CH}_{3} \mathrm{CN}, v_{8}=1$ and $v_{8}=2$ 


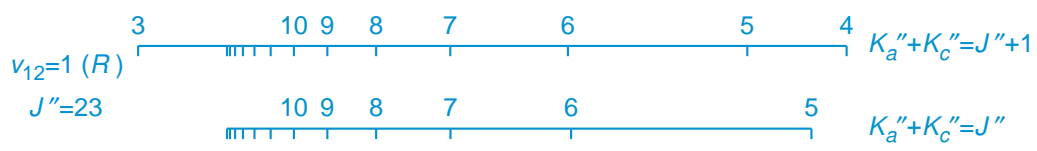

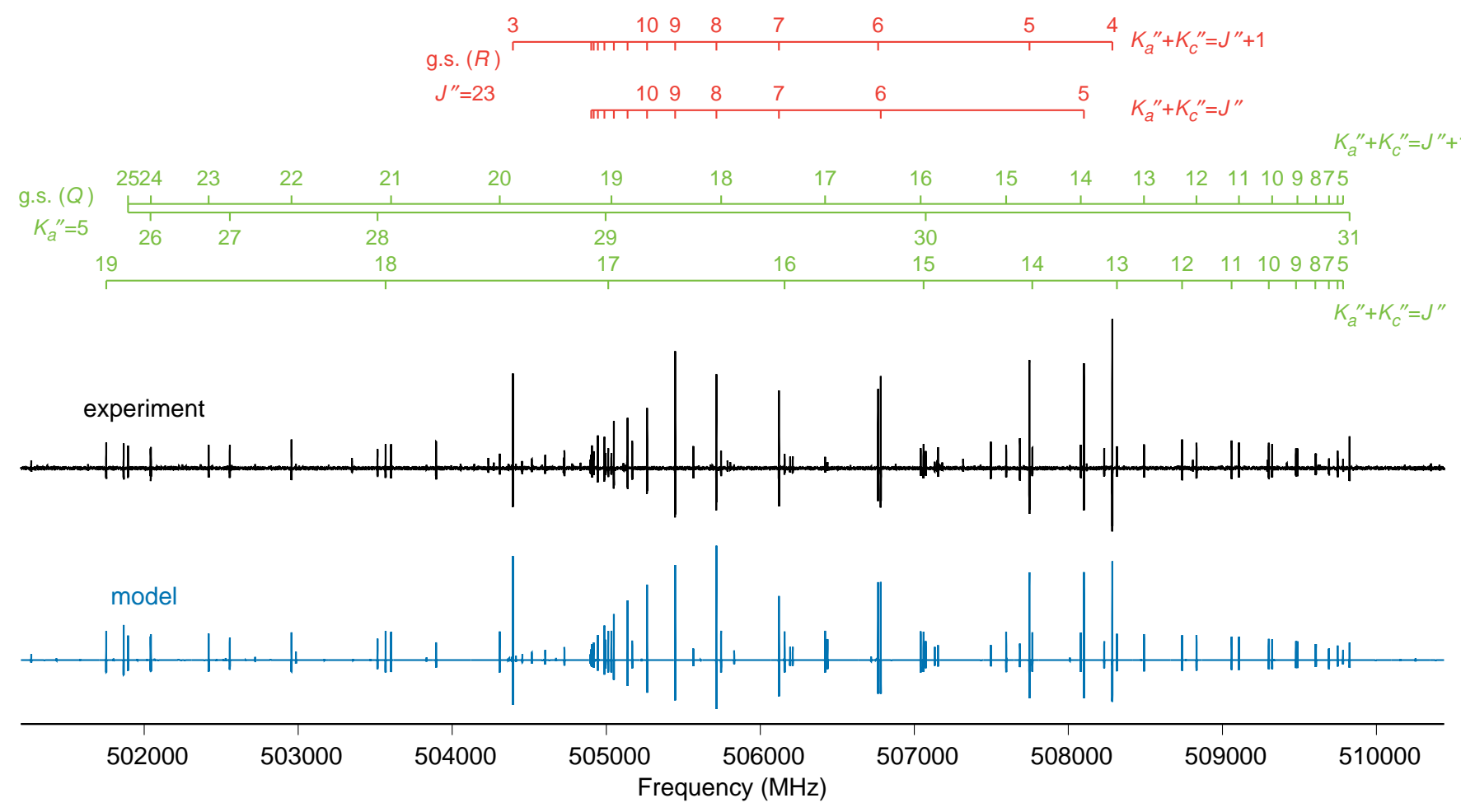

Fig. 1. Sample spectrum of $\mathrm{CH}_{2} \mathrm{NOH}$ at $500 \mathrm{GHz}$. The experimental spectrum is plotted in black, and the model (with both the ground state and the $v_{12}=1$ state) is plotted in blue sticks. Above the spectrum, the frequencies of three different branches are illustrated separately. Quantum numbers of the upper state are labeled for each branch where space is permitted.

vibrational excited states, and from ${ }^{13} \mathrm{CH}_{3} \mathrm{CN}, \mathrm{CH}_{3}{ }^{13} \mathrm{CN}$, and $\mathrm{CH}_{3} \mathrm{C}^{15} \mathrm{~N}$ isotopologues. The line lists from the CDMS catalog (Müller et al. 2005) were used to clearly flag out these lines. Although these lines are strong (even stronger than the lines of $\mathrm{CH}_{2} \mathrm{NOH}$ ), they are sparsely located in the spectrum and therefore do not pose serious difficulties in analyzing the transitions from $\mathrm{CH}_{2} \mathrm{NOH}$.

To guide the assignment of the millimeter- and submillimeter-wave spectrum of $\mathrm{CH}_{2} \mathrm{NOH}, 124$ previously reported microwave transitions (Levine 1962; Klesing \& Sutter 1990) were used to prepare a prediction for the ground state. Frequency uncertainties were assigned to lines in the literature based on their experimental methods $(0.1 \mathrm{MHz}$ for Levine 1962 and $2 \mathrm{kHz}$ for Klesing \& Sutter 1990). In this prediction, due to the limited number of $J$ and $K_{\mathrm{a}}$ levels accessed, only the rotational constants $A, B$, and $C$ and the quartic centrifugal distortion constants can be determined. Nevertheless, this prediction is sufficiently accurate to guide the assignment of our millimeter- and submillimeter-wave spectrum. The $a$-type, $R$ branch lines of $\mathrm{CH}_{2} \mathrm{NOH}$ are easily identified at a spacing of $B+C \approx 12 \mathrm{GHz}$. In addition, $b$-type, $Q$ branches can also be found. As expected, frequency shifts from the microwave prediction were observed up to $\sim 50 \mathrm{MHz}$, especially for high $J$ and $K_{\mathrm{a}}$ lines.

The $v_{12}=1$ state is the vibrational excited state of the lowest energy $\left(373 \mathrm{~cm}^{-1}, \mathrm{Wu} \& \operatorname{Tan} 2020\right)$. For this state, the microwave line list in the literature, with only four rotational transitions and nine observed hyperfine components in total (Levine 1962), is insufficient to produce a reliable prediction. Nevertheless, it is not difficult to find the vibrational satellites of approximately $16 \%$ of the intensity of the ground state lines. Once the vibrational satellites for the strongest $K_{\mathrm{a}}=0,1,2$ lines for a series of $J$ levels were identified, it was then possible to generate a preliminary fit with approximate rotational constants for the $v_{12}=1$ state, using identical centrifugal distortion constants obtained for the ground state. We used the preliminary fit to generate the prediction that was sufficiently accurate to guide the assignment of the rest of the $v_{12}=1$ state lines.

In the final assignment, 1433 millimeter- and submillimeterwave lines were assigned to 2108 unique transitions (hyperfine transitions included) for the ground state, and 921 millimeterand submillimeter-wave lines were assigned to 1298 unique transitions (hyperfine transitions included) for the $v_{12}=1$ state. The highest $J, K_{\mathrm{a}}$, and $K_{\mathrm{c}}$ accessed are 50, 24, and 44, respectively, for the ground state, and 45, 18, and 40, respectively, for the $v_{12}=1$ state. The microwave lines were also included for the analysis. The full list of the measured transitions is summarized in Tables 2 and 3. An $8 \mathrm{GHz}$ wide example of the $\mathrm{CH}_{2} \mathrm{NOH}$ spectrum is plotted in Fig. 1. In this example, we can clearly identify the $a$-type, $R$ branch for the ground state and the vibrationally excited state, with the upper state $J^{\prime \prime}=23$, and $K_{\mathrm{a}}^{\prime \prime}=3-16$. In addition to the $R$ branches, the $b$-type, $Q$ branch of the ground state is also present, with the upper state $K_{\mathrm{a}}^{\prime \prime}=5$, and $J^{\prime \prime}=5-31$. We used the ab initio dipole moment, $0.26 \mathrm{D}$ for $a$-dipole and 
Table 2. Measured frequencies and fit residual of formaldoxime in the ground state.

\begin{tabular}{|c|c|c|c|c|c|c|c|c|c|c|c|c|c|}
\hline \multicolumn{5}{|c|}{ Upper state } & \multicolumn{5}{|c|}{ Lower state } & \multirow{2}{*}{$\begin{array}{c}\text { Frequency } \\
(\mathrm{MHz})\end{array}$} & \multirow{2}{*}{$\begin{array}{c}\text { Relative } \\
\text { Intensity }\end{array}$} & \multirow{2}{*}{$\begin{array}{c}\text { obs. }- \text { calc. }{ }^{(3)} \\
(\mathrm{MHz})\end{array}$} & \multirow{2}{*}{$\begin{array}{c}\text { Uncertainty } \\
(\mathrm{MHz})\end{array}$} \\
\hline$J^{\prime \prime}$ & $K_{\mathrm{a}}^{\prime \prime}$ & $K_{\mathrm{c}}^{\prime \prime}$ & $v_{\text {dummy }}^{\prime \prime}(1)$ & $F^{\prime \prime}$ & $J^{\prime}$ & $K_{\mathrm{a}}^{\prime}$ & $K_{\mathrm{c}}^{\prime}$ & $v_{\text {dummy }}^{\prime}{ }^{(1)}$ & $F^{\prime}$ & & & & \\
\hline 7 & 2 & 5 & 1 & 7 & 7 & 1 & 6 & 1 & 7 & 150977.130 & 1.00 & -0.00976 & 0.050 \\
\hline 7 & 2 & 5 & 1 & 6 & 7 & 1 & 6 & 1 & 6 & 150977.948 & 0.43 & -0.07800 & 0.050 \\
\hline 7 & 2 & 5 & 1 & 8 & 7 & 1 & 6 & 1 & 8 & 150977.948 & 0.57 & 0.03358 & 0.050 \\
\hline 12 & 1 & 11 & 1 & 11 & 12 & 0 & 12 & 1 & 11 & 150093.192 & 1.00 & 0.08233 & 0.050 \\
\hline 12 & 1 & 11 & 1 & 13 & 12 & 0 & 12 & 1 & 13 & 150093.192 & 1.00 & -0.06927 & 0.050 \\
\hline 12 & 1 & 11 & 1 & 12 & 12 & 0 & 12 & 1 & 12 & 150095.057 & 1.00 & -0.01538 & 0.050 \\
\hline 7 & 0 & 7 & 0 & 7 & 6 & 0 & 6 & 0 & 6 & 150292.461 & 1.00 & 0.00868 & 0.030 \\
\hline 10 & 4 & 7 & 1 & 9 & 11 & 3 & 8 & 1 & 10 & 152004.918 & 0.45 & -0.01526 & 0.060 \\
\hline 10 & 4 & 7 & 1 & 11 & 11 & 3 & 8 & 1 & 12 & 152004.918 & 0.55 & 0.01425 & 0.060 \\
\hline 7 & 2 & 6 & 0 & 7 & 6 & 2 & 5 & 0 & 6 & 153238.987 & 1.00 & -0.00144 & 0.050 \\
\hline
\end{tabular}

Notes. ${ }^{(1)}$ The dummy vibrational quantum number $v_{\text {dummy }}$ labels the unresolved $\left(v_{\text {dummy }}=0\right)$ and resolved $\left(v_{\text {dummy }}=1\right)$ hyperfine splitting. For $v_{\text {dummy }}=0$ lines, $F \equiv J .{ }^{(2)}$ For single lines, the relative intensity is always unity. For blended lines, the sum of the relative intensities is unity. ${ }^{(3)}$ Frequency calculated using the $S$-reduction. Full table available at the CDS.

Table 3. Measured frequencies and fit residual of formaldoxime in the $v_{12}=1$ state.

\begin{tabular}{|c|c|c|c|c|c|c|c|c|c|c|c|c|c|}
\hline \multicolumn{5}{|c|}{ Upper state } & \multicolumn{5}{|c|}{ Lower state } & \multirow{2}{*}{$\begin{array}{c}\text { Frequency } \\
(\mathrm{MHz})\end{array}$} & \multirow{2}{*}{$\begin{array}{c}\text { Relative } \\
\text { intensity }^{(2)}\end{array}$} & \multirow{2}{*}{$\begin{array}{c}\text { obs. - calc. } \\
(\mathrm{MHz})\end{array}$} & \multirow{2}{*}{$\begin{array}{c}\text { Uncertainty } \\
(\mathrm{MHz})\end{array}$} \\
\hline$J^{\prime \prime}$ & $K_{\mathrm{a}}^{\prime \prime}$ & $K_{\mathrm{c}}^{\prime \prime}$ & $v_{\text {dummy }}^{\prime \prime}$ & $F^{\prime \prime}$ & $J^{\prime}$ & $K_{\mathrm{a}}^{\prime}$ & $K_{\mathrm{c}}^{\prime}$ & $v_{\text {dummy }}^{\prime}(1)$ & $F^{\prime}$ & & & & \\
\hline 7 & 0 & 7 & 0 & 7 & 6 & 0 & 6 & 0 & 6 & 150194.364 & 1.00 & -0.00237 & 0.050 \\
\hline 15 & 2 & 13 & 1 & 16 & 15 & 1 & 14 & 1 & 16 & 150460.786 & 0.53 & -0.04598 & 0.050 \\
\hline 15 & 2 & 13 & 1 & 14 & 15 & 1 & 14 & 1 & 14 & 150460.786 & 0.47 & -0.01023 & 0.050 \\
\hline 15 & 2 & 13 & 1 & 15 & 15 & 1 & 14 & 1 & 15 & 150461.334 & 1.00 & -0.03328 & 0.050 \\
\hline 7 & 2 & 6 & 0 & 7 & 6 & 2 & 5 & 0 & 6 & 153094.237 & 1.00 & 0.00921 & 0.050 \\
\hline 7 & 5 & 2 & 1 & 6 & 6 & 5 & 1 & 1 & 5 & 153563.055 & 0.21 & 0.02746 & 0.050 \\
\hline 7 & 5 & 2 & 1 & 8 & 6 & 5 & 1 & 1 & 7 & 153563.055 & 0.29 & -0.06205 & 0.050 \\
\hline 7 & 5 & 3 & 1 & 8 & 6 & 5 & 2 & 1 & 7 & 153563.055 & 0.29 & -0.05765 & 0.050 \\
\hline 7 & 5 & 3 & 1 & 6 & 6 & 5 & 2 & 1 & 5 & 153563.055 & 0.21 & 0.03186 & 0.050 \\
\hline 7 & 5 & 3 & 1 & 7 & 6 & 5 & 2 & 1 & 6 & 153563.585 & 0.50 & -0.00873 & 0.050 \\
\hline 7 & 5 & 2 & 1 & 7 & 6 & 5 & 1 & 1 & 6 & 153563.585 & 0.50 & -0.01313 & 0.050 \\
\hline
\end{tabular}

Notes. ${ }^{(1)}$ The dummy vibrational quantum number $v_{\text {dummy }}$ labels the unresolved $\left(v_{\text {dummy }}=0\right)$ and resolved $\left(v_{\text {dummy }}=1\right)$ hyperfine splitting. For $v_{\text {dummy }}=0$ lines, $F \equiv J .{ }^{(2)}$ For single lines, the relative intensity is always unity. For blended lines, the sum of the relative intensities is unity. ${ }^{(3)}$ Frequency calculated using the $S$-reduction. Full table available at the CDS.

$0.19 \mathrm{D}$ for $b$-dipole, to generate the overall prediction, because we found that the relative intensities between the $a$-type and $b$-type lines are better reproduced using the ab initio values, compared with the experimental dipole moment reported by Levine (1962). Figure 1 demonstrates an excellent agreement between the prediction and the experimental spectrum.

The analysis of the ground state and $v_{12}=1$ state was conducted separately because no obvious interaction between the two states was observed. A large portion of our measured transitions shows at least partially resolved hyperfine splitting due to the ${ }^{14} \mathrm{~N}$ atom. To fit all transitions together, we used a "dummy" vibrational state quantum number in the SPFIT program to label the hyperfine lines. The spectroscopic parameters in the rotational Hamiltonian were shared across the states, while the hyperfine splitting parameters were associated only to the hyperfine state. Except for a few $J^{\prime \prime} \leq 3$ lines, we could only assign the three strong $\Delta F=\Delta J$ hyperfine components to partially resolved hyperfine splitting lines because the intensity of other $\Delta F \neq \Delta J$ components scales with $1 /\left(2 J^{2}\right)$ and is below the detection limit. Therefore, the off-diagonal element $\chi_{a b}$ in the quadrupole hyperfine tensor cannot be determined during the fit. We fixed it to the ab initio calculation value.
For the rotational Hamiltonian, the Watson's $S$-reduction is presumably preferred for $\mathrm{CH}_{2} \mathrm{NOH}$, because it is a nearly symmetric prolate-top rotor (Ray's asymmetry parameter $\kappa=$ -0.938), which was discussed in the work of dimethylsulfoxide (Margulès et al. 2010). The $s_{111}$ parameter for $\mathrm{CH}_{2} \mathrm{NOH}$ is $3.4 \times 10^{-7}$ for $A$-reduction, and $-6.0 \times 10^{-8}$ for $S$-reduction. Nevertheless, for comparison, we used both the Watson's $A$-reduction and $S$-reduction Hamiltonian for the fit. In order to fully describe the Hamiltonian, centrifugal distortion constants higher than the fourth order are necessary. For both the $A$-reduction and $S$-reduction Hamiltonian, we first introduced all seven sextic centrifugal distortion constants into the fit of the ground state lines. All of these terms were statistically significant, and the r.m.s. of the fit result $\sigma$ was reduced to $83.3 \mathrm{kHz}$, which is still larger than our experimental accuracy of the frequency measurements, being approximately $50 \mathrm{kHz}$. The weighted r.m.s. $\sigma_{\mathrm{w}}$ is 1.43 , which implies that statistically significant effects are missing in the current model. Therefore, octic and decic constants were furthermore introduced to the fit, and only those with statistical significance were chosen to produce the final fit with $\sigma=32.4 \mathrm{kHz}$ and weighted $\sigma_{w}=0.81$ both satisfactory to our experimental uncertainty. The similar procedure 
Table 4. Spectroscopic constants of $\mathrm{CH}_{2} \mathrm{NOH}, S$-reduction.

\begin{tabular}{|c|c|c|c|c|}
\hline \multirow[t]{2}{*}{ Parameters (MHz) } & \multicolumn{2}{|c|}{ Ground state } & \multicolumn{2}{|c|}{$v_{12}=1$ state } \\
\hline & This work & Ref. ${ }^{(5)}$ & This work & Ref. ${ }^{(5)}$ \\
\hline$A$ & $67679.40866(67)^{(1)}$ & $67679.389(13)$ & $66634.0765(26)$ & $66634.045(12)$ \\
\hline$B$ & $11859.22541(10)$ & $11859.2158(25)$ & $11829.50007(23)$ & $11829.506(12)$ \\
\hline$C$ & $10080.12451(10)$ & $10080.1194(27)$ & $10088.09344(23)$ & $10088.049(16)$ \\
\hline$D_{J} \times 10^{3}$ & $9.41688(15)$ & $9.3922(28)$ & $9.55112(41)$ & $9.5247(24)$ \\
\hline$D_{J K}$ & $-0.0247399(15)$ & $-0.024562(28)$ & $-0.0238304(61)$ & $-0.023902(25)$ \\
\hline$D_{K}$ & $1.424516(19)$ & $0.014237(94)$ & $1.26957(18)$ & $1.26454(13)$ \\
\hline$d_{1} \times 10^{3}$ & $-1.903093(27)$ & $-1.8980(61)$ & $-1.878438(98)$ & $-1.8650(75)$ \\
\hline$d_{2} \times 10^{3}$ & $-0.182984(18)$ & $-0.1862(18)$ & $-0.117691(36)$ & $-0.1508(55)$ \\
\hline$H_{J} \times 10^{9}$ & $5.051(72)$ & & $5.67(25)$ & \\
\hline$H_{J K} \times 10^{6}$ & $-0.26567(65)$ & $-23.110(15)$ & $-0.6009(46)$ & $-5.964(11)$ \\
\hline$H_{K J} \times 10^{6}$ & $-8.2687(73)$ & $-7.831(88)$ & $-8.75(11)$ & $-8.430(99)$ \\
\hline$H_{K} \times 10^{3}$ & $0.09206(19)$ & $0.08775(27)$ & $0.2919(47)$ & $0.03975(40)$ \\
\hline$h_{1} \times 10^{9}$ & 3.784(18) & & $4.248(57)$ & \\
\hline$h_{2} \times 10^{9}$ & $1.065(15)$ & & $0.548(33)$ & \\
\hline$h_{3} \times 10^{9}$ & $0.3595(35)$ & & $-0.6867(91)$ & \\
\hline$L_{J K} \times 10^{9}$ & & & $2.703(74)$ & \\
\hline$L_{K K J} \times 10^{9}$ & $0.740(23)$ & & $5.12(54)$ & \\
\hline$L_{K} \times 10^{6}$ & $-0.00800(57)$ & & $-3.563(32)$ & \\
\hline$P_{K J} \times 10^{12}$ & $-0.072(13)$ & & $-6.20(22)$ & \\
\hline$P_{K K J} \times 10^{9}$ & & & $-0.01275(96)$ & \\
\hline$\chi_{a a}$ & $2.9870(16)$ & & $2.445(77)$ & \\
\hline$\chi_{b b}$ & $-4.6838(14)$ & & $-5.321(51)$ & \\
\hline$\chi_{a b}$ & $3.6806638^{(2)}$ & & $3.6806638^{(2)}$ & \\
\hline$\sigma^{(3)}$ & 0.0324 & & 0.0469 & \\
\hline$\sigma_{w}^{(4)}$ & 0.81 & & 0.83 & \\
\hline
\end{tabular}

Notes. ${ }^{(1)}$ The $1-\sigma$ uncertainty is displayed in the parentheses in the unit of the last digit. ${ }^{(2)}$ Fixed to B3PW91/6-311+G(df,pd) calculation value. ${ }^{(3)}$ r.m.s. deviation of the fit. ${ }^{(4)}$ Weighted r.m.s. deviation of the fit.

References. ${ }^{(5)} \mathrm{Wu} \&$ Tan (2020), values converted from wavenumber to megahertz.

was performed on the $v_{12}=1$ lines, and the final fit produces a $\sigma$ of $46.9 \mathrm{kHz}$ and $\sigma_{\mathrm{w}}$ of 0.83 , which again agree well with our experimental uncertainty. The full list of the spectroscopic constants of the ground state and the $v_{12}=1$ state is shown in Tables 4 and 5, respectively.

In general, the spectroscopic constants obtained by the Watson's $A$-reduction and $S$-reduction Hamiltonian are of a similar accuracy. However, several sextic centrifugal distortion constants in the $S$-reduction are slightly better determined than those in the $A$-reduction. These constants include $\Phi_{J K}, \Phi_{K J}$, and $\phi_{J K}$ in the $A$-reduction and $H_{J K}, H_{K J}$, and $h_{2}$ in the $S$-reduction. For the ground state fit, the relative uncertainty of these constants in the $S$-reduction is one order of magnitude lower than those in the $A$-reduction. For the $v_{12}=1$ state fit, $\phi_{J K}$ cannot be statistically determined, whereas $h_{2}$ can. The absolute values of $\delta_{K}, \phi_{J K}$, and $\phi_{K}$ in the $A$-reduction are 2-4 orders of magnitude larger than the corresponding $d_{2}, h_{2}$, and $h_{3}$ terms in the $S$-reduction. This is due to the different constraints applied to the sextic terms of the angular momentum operators, and the constraint applied by the $S$-reduction is more suitable for the nearly symmetric top case where $B-C$ is small. As such, the $S$-reduction fit is preferred when generating the millimeter- and submillimeter-wave prediction of $\mathrm{CH}_{2} \mathrm{NOH}$.

A combination fit of the microwave, millimeter- and submillimeter-wave, and infrared data for the ground state was tested using the $S$-reduction Hamiltonian. The weighted r.m.s. $\sigma_{\mathrm{w}}$ grew to 2.6, which is significantly higher than the fit with only microwave, millimeter- and submillimeter-wave data. All spectroscopic constants agree with the values in Table 4 within statistical uncertainty. Due to the relatively large experimental uncertainty, the weight on each infrared line is $<1 / 3600$ compared to each millimeter- and submillimeter-wave line. Because the number of lines and the access of quantum numbers of the two data sets are similar, the infrared data have no significant impact on the fit results.

A large discrepancy was found for the ground state $D_{K}$ term in the $S$-reduction between our result and that from $\mathrm{Wu} \&$ Tan (2020), but not for the $v_{12}=1$ state. Among the four $D_{K}$ values in the $S$-reduction fit of this work and Wu \& Tan (2020) for both states, and the four corresponding $\Delta_{K}$ values in the $A$-reduction fit, the ground state $D_{K}$ term in Wu \& Tan (2020) is the only outlier and two orders of magnitude smaller than all other seven values. It is also two orders of magnitude smaller than the value reported in Wu \& Tan (2021), where more lines were used for the ground state fit. Since $D_{K}$ and $\Delta_{K}$ correspond to the same angular momentum operator $J_{z}^{4}$, their values should be similar regardless of the reduction chosen. The ground state $D_{K}$ value reported by Wu \& Tan (2020) is probably a typographical error.

Thanks to the higher frequency resolution and the access to higher $J$ and $K_{\mathrm{a}}$ quantum numbers, our spectroscopic constants for both the ground state and $v_{12}=1$ state are in general one to two orders of magnitude more accurate than the result from the 
Table 5. Spectroscopic constants of $\mathrm{CH}_{2} \mathrm{NOH}, A$-reduction.

\begin{tabular}{|c|c|c|c|c|}
\hline \multirow[t]{2}{*}{ Parameters (MHz) } & \multicolumn{2}{|c|}{ Ground state } & \multicolumn{2}{|c|}{$v_{12}=1$ state } \\
\hline & This work & Ref. ${ }^{(5)}$ & This work & Ref. $^{(5)}$ \\
\hline$A$ & $67679.40610(67)^{(1)}$ & $67679.386(13)$ & $66634.0764(25)$ & $66634.018(12)$ \\
\hline$B$ & $11859.31955(10)$ & $11859.3109(27)$ & $11829.56055(23)$ & $11829.585(13)$ \\
\hline$C$ & $10080.03162(10)$ & $10080.0240(24)$ & $10088.03414(23)$ & $10087.971(16)$ \\
\hline$\Delta_{J} \times 10^{3}$ & $9.78269(15)$ & $9.7612(58)$ & $9.78670(41)$ & $9.822(12)$ \\
\hline$\Delta_{J K}$ & $-0.0269398(15)$ & $-0.026780(40)$ & $-0.0252413(60)$ & $-0.025689(82)$ \\
\hline$\Delta_{K}$ & $1.426345(19)$ & $1.42556(10)$ & $1.27079(18)$ & $1.26602(16)$ \\
\hline$\delta_{J} \times 10^{3}$ & $1.903150(25)$ & $1.8992(61)$ & $1.878529(88)$ & $1.8677(76)$ \\
\hline$\delta_{K} \times 10^{3}$ & $46.7717(40)$ & $47.478(46)$ & $29.8874(46)$ & $38.4(14)$ \\
\hline$\Phi_{J} \times 10^{9}$ & $7.118(80)$ & & $6.85(25)$ & \\
\hline$\Phi_{J K} \times 10^{6}$ & $0.0880(39)$ & $-0.228(15)$ & $-1.3043(87)$ & $-0.602(11)$ \\
\hline$\Phi_{K J} \times 10^{6}$ & $-9.521(15)$ & $-7.834(90)$ & $-6.42(11)$ & $-8.40(10)$ \\
\hline$\Phi_{K} \times 10^{3}$ & $0.09295(19)$ & $0.08769(27)$ & $0.2913(46)$ & $0.03969(40)$ \\
\hline$\phi_{J} \times 10^{9}$ & $4.146(20)$ & & $3.634(48)$ & \\
\hline$\phi_{J K} \times 10^{9}$ & $0.0497(39)$ & & & \\
\hline$\phi_{K} \times 10^{3}$ & $0.01578(16)$ & & $-0.02972(32)$ & \\
\hline$L_{J K} \times 10^{9}$ & & & $2.752(74)$ & \\
\hline$L_{K K J} \times 10^{9}$ & $0.820(23)$ & & $5.18(54)$ & \\
\hline$L_{K} \times 10^{6}$ & $-0.00808(57)$ & & $-3.571(32)$ & \\
\hline$P_{K J} \times 10^{12}$ & $-0.091(13)$ & & $-6.77(22)$ & \\
\hline$P_{K K J} \times 10^{9}$ & & & $-0.01178(95)$ & \\
\hline$\chi_{a a}$ & $2.9870(16)$ & & $2.451(77)$ & \\
\hline$\chi_{b b}$ & $-4.6838(14)$ & & $-5.323(51)$ & \\
\hline$\chi_{a b}$ & $3.6806638^{(2)}$ & & $3.6806638^{(2)}$ & \\
\hline$\overline{\sigma^{(3)}}$ & 0.0328 & & 0.0469 & \\
\hline$\sigma_{w}^{(4)}$ & 0.81 & & 0.83 & \\
\hline
\end{tabular}

Notes. ${ }^{(1)}$ The $1-\sigma$ uncertainty is displayed in the parentheses in the unit of the last digit. ${ }^{(2)}$ Fixed to B3PW91/6-311+G(df,pd) calculation value. ${ }^{(3)}$ r.m.s. deviation of the fit. ${ }^{(4)}$ Weighted r.m.s. deviation of the fit.

References. ${ }^{(5)} \mathrm{Wu} \& \mathrm{Tan}(2020)$, values converted from wavenumber to megahertz.

synchrotron infrared measurement (Wu \& Tan 2020). Using our spectroscopic constants shown in Tables 4 and 5, we can generate the line catalog prediction of $\mathrm{CH}_{2} \mathrm{NOH}$ up to $J=50$ and $1 \mathrm{THz}$. In the line catalog, we removed the "dummy" quantum number that we used to label the hyperfine resolved lines, and we merged the predictions of the ground state and the $v_{12}=1$ state. Because of the strong centrifugal distortion effects of $\mathrm{CH}_{2} \mathrm{NOH}$, the estimated uncertainty of the predicted frequencies can exceed $1 \mathrm{MHz}$ for quantum numbers that we were unable to measure. These predicted frequencies need to be treated with caution. The predictions are listed in Tables A.1 and A.2. Both tables can also be found in the supporting material CDS: S3-S4.

\section{Implication to astronomy}

The presence of $\mathrm{CH}_{2} \mathrm{NOH}$ in the $\mathrm{N}$ astrochemistry network has been long postulated. The $\mathrm{NH}_{2} \mathrm{CHO} / \mathrm{CH}_{2} \mathrm{NOH}$ ratio is estimated to range from 11.4:1 to 4:1 in dark clouds (Allen \& Robinson 1977). Although $\mathrm{NH}_{2} \mathrm{CHO}$ is commonly observed in multiple sources, no positive detection of $\mathrm{CH}_{2} \mathrm{NOH}$ has been claimed to date. In addition to the lower number density of $\mathrm{CH}_{2} \mathrm{NOH}$, the dipole moment of $\mathrm{CH}_{2} \mathrm{NOH}$ (0.32 Debye) is significantly smaller than the dipole moment of $\mathrm{NH}_{2} \mathrm{CHO}$ (3.6 Debye) (Kurland \& Wilson 1957). As a result, if we assume the $\mathrm{NH}_{2} \mathrm{CHO} / \mathrm{CH}_{2} \mathrm{NOH}$ ratio to be $10: 1$, the line intensity of $\mathrm{CH}_{2} \mathrm{NOH}$ would be three orders of magnitude weaker than $\mathrm{NH}_{2} \mathrm{CHO}$. Therefore, the search for $\mathrm{CH}_{2} \mathrm{NOH}$, if it does exist in the ISM, requires sensitive observations toward ideal sources where nonthermal processes are able to drive a considerable amount of $\mathrm{CH}_{2} \mathrm{NOH}$ into the gas phase.

\section{Conclusion}

In conclusion, we have measured the millimeter- and submillimeter-wave lines of $\mathrm{CH}_{2} \mathrm{NOH}$ in the range of 150 $660 \mathrm{GHz}$ using direct absorption spectroscopy, and we have obtained the most accurate fit to date for both the ground state and the $v_{12}=1$ vibrationally excited state. Both Watson's $A$ reduction and $S$-reduction Hamiltonian were used to fit the transitions, and we determined that the $S$-reduction is more suitable for $\mathrm{CH}_{2} \mathrm{NOH}$. A prediction for the $\mathrm{CH}_{2} \mathrm{NOH}$ line list up to $1 \mathrm{THz}$ is provided using our fit spectroscopic constants.

Acknowledgements. This work has been supported by the "Programme National Physique et Chimie du Milieu Interstellaire" (PCMI) of CNRS/INSU with INC/INP co-funded by CEA and CNES, and by the French ANR Labex CaPPA through the PIA under Contract no. ANR-11-LABX-0005-01, the Regional Council of Hauts-de-France, the European Funds for Regional Economic Development (FEDER), and the contract CPER CLIMIBIO. L.Z. thanks the financial support from the European Union's Horizon 2020 research and innovation programme under the Marie Skłodowska-Curie Individual Fellowship grant (H2020-MSCA-IF-2019, Project no. 894508). J.C.G. thanks the Centre National d'Etudes Spatiales (CNES) for a grant. 


\section{References}

Allen, M., \& Robinson, G. W. 1977, ApJ, 212, 396

Bannai, R. A., \& Duxbury, G. 1994, J. Opt. Soc. Am. B, 11, 170

Dunstan, W. R., \& Bossi, A. L. 1898, J. Chem. Soc. Trans., 73, 353

Duxbury, G. 1988, J. Mol. Spectr., 132, 393

Duxbury, G., \& Petersen, J. 1984, Appl. Phys. B, 35, 127

Duxbury, G., Percival, R., Devoy, D., \& Mahmoud, M. 1988, J. Mol. Spectr. 132, 380

Frisch, M. J., Trucks, G. W., Schlegel, H. B., et al. 2016, Gaussian 09, Revision B.01

Godfrey, P. D., Brown, R. D., Robinson, B. J., \& Sinclair, M. W. 1973, ApJ, 13, L119

Herbst, E. \& van Dishoeck, E. F. 2009, ARA\&A, 47, 427

Kaushik, V. K., \& Takagi, K. 1978, J. Phys. Soc. Japan, 45, 1975

Klesing, A., \& Sutter, D. H. 1990, Z. Naturforsch. A, 45, 817

Kurland, R. J., \& Wilson, E. B. 1957, J. Chem. Phys., 27, 585

Lattelais, M., Pauzat, F., Ellinger, Y., \& Ceccarelli, C. 2010, A\&A, 519, A30

Levine, I. N. 1962, J. Mol. Spectr., 8, 276

Levine, I. N. 1963, J. Chem. Phys., 38, 2326
Margulès, L., Motiyenko, R., Alekseev, E., \& Demaison, J. 2010, J. Mol. Spectr., 260, 23

McGuire, B. A. 2018, ApJS, 239, 17

Motiyenko, R. A., Armieieva, I. A., Margulès, L., Alekseev, E. A., \& Guillemin, J.-C. 2019, A\&A, 623, A162

Müller, H., Schlöder, F., Stutzki, J., \& Winnewisser, G. 2005, J. Mol. Struct., 742,215

Pickett, H. M. 1991, J. Mol. Spectr., 148, 371

Pickett, H. M., Poynter, R. L., Cohen, E. A., et al. 1998, J. Quant. Spectr. Rad. Trans., 60, 883

Pillai, M. G. K. 1962, J. Phys. Chem., 66, 179

Rivilla, V. M., Martín-Pintado, J., Jiménez-Serra, I., et al. 2020, ApJ, 899, L28

Rubin, R. H., Swenson, Jr. G. W., Benson, R. C., Tigelaar, H. L., \& Flygare, W. H. 1971, ApJ, 169, L39

Umar, Y., Jimoh, T., \& Morsy, M. 2005, J. Mol. Struct.: Theochem., 725, 157

Vinogradoff, V., Duvernay, F., Danger, G., Theulé, P., \& Chiavassa, T. 2011, A\&A, 530, A128

Wu, Q., \& Tan, T. 2021, J. Mol. Spectr., 376, 111417

Wu, Q. Y., \& Tan, T. L. 2020, J. Mol. Spectr., 111290

Zakharenko, O., Motiyenko, R. A., Margulès, L., \& Huet, T. R. 2015, J. Mol. Spectr., 317, 41 


\section{Appendix A: Predictions of $\mathrm{CH}_{2} \mathrm{NOH}$ lines}

Table A.1. Prediction of pure rotational formaldoxime transitions up to $1 \mathrm{THz}$ for both the ground state and $v_{12}=1$ state.

\begin{tabular}{ccccrrrrrrrrrrrrr}
\hline \hline FREQ & UNC & LOGINT & DR & ELO & GUP & TAG & QNFMT & $J^{\prime \prime}$ & $K_{\mathrm{a}}^{\prime \prime}$ & $K_{\mathrm{c}}^{\prime \prime}$ & $v^{\prime \prime}$ & $J^{\prime}$ & $K_{\mathrm{a}}^{\prime}$ & $K_{\mathrm{c}}^{\prime}$ & $v^{\prime}$ \\
\hline 1455.7529 & 0.0002 & -9.9476 & 3 & 18.5387 & 11 & 45999 & 1404 & 5 & 2 & 3 & 0 & 5 & 2 & 4 & 0 \\
1779.0857 & 0.0001 & -9.7278 & 3 & 2.5937 & 3 & 45999 & 1404 & 1 & 1 & 0 & 0 & 1 & 1 & 1 & 0 \\
1805.7069 & 0.0005 & -9.8340 & 3 & 65.4276 & 23 & 45999 & 1404 & 11 & 3 & 8 & 0 & 11 & 3 & 9 & 0 \\
1966.5414 & 0.0080 & -9.9552 & 3 & 142.5542 & 35 & 45999 & 1404 & 17 & 4 & 13 & 0 & 18 & 3 & 16 & 0 \\
\hline
\end{tabular}

Notes. Full table available at the CDS.

Table A.2. Prediction of pure rotational formaldoxime transitions including ${ }^{14} \mathrm{~N}$ hyperfine components up to $1 \mathrm{THz}$ for both the ground state and $v_{12}=1$ state.

\begin{tabular}{ccccrrrrrrrrrrrrrrrrrrr}
\hline \hline FREQ & UNC & LOGINT & DR & ELO & GUP & TAG & QNFMT & $J^{\prime \prime}$ & $K_{\mathrm{a}}^{\prime \prime}$ & $K_{\mathrm{c}}^{\prime \prime}$ & $v^{\prime \prime}$ & $F^{\prime \prime}$ & $J^{\prime}$ & $K_{\mathrm{a}}^{\prime}$ & $K_{\mathrm{c}}^{\prime}$ & $v^{\prime}$ & $F^{\prime}$ \\
\hline 4590.4839 & 0.0027 & -9.8863 & 3 & 83.4114 & 25 & 45999 & 1405 & 13 & 3 & 10 & 1 & 12 & 13 & 3 & 11 & 1 & 12 \\
4590.4839 & 0.0027 & -9.8551 & 3 & 83.4114 & 27 & 45999 & 1405 & 13 & 3 & 10 & 1 & 13 & 13 & 3 & 11 & 1 & 13 \\
4590.4839 & 0.0027 & -9.8215 & 3 & 83.4114 & 29 & 45999 & 1405 & 13 & 3 & 10 & 1 & 14 & 13 & 3 & 11 & 1 & 14 \\
4671.3988 & 0.0141 & -9.9712 & 3 & 233.0298 & 49 & 45999 & 1405 & 23 & 4 & 20 & 0 & 24 & 22 & 5 & 17 & 0 & 23 \\
4671.4936 & 0.0141 & -9.9902 & 3 & 233.0299 & 47 & 45999 & 1405 & 23 & 4 & 20 & 0 & 23 & 22 & 5 & 17 & 0 & 22 \\
\hline
\end{tabular}

Notes. Full table available at the CDS.

The prediction of $\mathrm{CH}_{2} \mathrm{NOH}$ lines using the Watson's $S$ reduction Hamiltonian up to $1 \mathrm{THz}$ is listed in Tables A.1 and A.2, which include the quadrupole hyperfine splitting components due to ${ }^{14} \mathrm{~N}$. Tables are listed using the JPL/CDMS molecular catalog format (Pickett et al. 1998). For both predictions, the maximum $J$ is 50 , and the minimum intensity $\left(\log _{10}\right.$ base) is -10.0 .

\section{Appendix B: Ab initio calculation}

The $x, y, z$ coordinates of $\mathrm{CH}_{2} \mathrm{NOH}$ after geometry optimization are listed in Table B.1.
Table B.1. Coordinates of $\mathrm{CH}_{2} \mathrm{NOH}$ after geometry optimization using B3LYP/6-311++g(3df,2pd). Unit is in $\AA$.

\begin{tabular}{crrr}
\hline \hline Atom & $x$ & $y$ & $z$ \\
\hline $\mathrm{C}$ & 1.146252 & -0.232496 & 0.000000 \\
$\mathrm{~N}$ & 0.123230 & 0.511091 & 0.000000 \\
$\mathrm{O}$ & -1.059067 & -0.234422 & 0.000000 \\
$\mathrm{H}$ & -1.744652 & 0.440282 & 0.000000 \\
$\mathrm{H}$ & 2.105860 & 0.264793 & 0.000000 \\
$\mathrm{H}$ & 1.086526 & -1.317611 & 0.000000 \\
\hline
\end{tabular}

